\title{
Cortical Processing during Dynamic Motor Adaptation
}

\author{
Simon A. Overduin, Andrew G. Richardson, and Emilio Bizzi
}

\begin{abstract}
In this chapter we investigate the role of motor cortex in adapting movements to novel dynamic environments. We present results from two experiments in which monkey or human subjects learned to make two-dimensional reaching movements while holding a robotic manipulandum that applied a predictable pattern of forces (a curl field) to their hand. In the first study, we analyzed electrophysiological data recorded in motor cortex while monkeys adapted or readapted to the novel forces on each day of the experiment. In the second study, we perturbed the excitability of motor cortex using repetitive transcranial magnetic stimulation (rTMS) as human participants adapted to the forces. From the first experiment, we present qualitative evidence that a network of cortical areas including the supplementary motor area, premotor cortex, and primary motor cortex (M1) not only encodes kinematic and dynamic parameters of motor execution, but also registers changes in encoding that could provide a substrate for motor memory. Based on the second experiment, we qualify the role of M1 in motor memory, by showing that its disruption by rTMS does not interfere with the process of initial motor adaptation, but rather with offline improvement as measured at retest on the following day.
\end{abstract}

\section{Introduction}

We routinely produce movements under different mechanical contexts. Behaviors such as swinging a hammer, opening a door, and lifting a carton of milk all involve forces acting on the moving arm that are not present when the arm is moving freely. Indeed, all interactions with the physical environment alter the forces experienced during a movement, or in other words, alter the movement dynamics. The dynamics of movement are governed by

\footnotetext{
S.A. Overduin $(\bowtie)$

Department of Brain and Cognitive Sciences and McGovern Institute for Brain Research, Massachusetts Institute of Technology, 43 Vassar Street, Cambridge, MA 02139, USA

e-mail: overduin@mit.edu
} 
constitutive mechanical properties (e.g. stiffness, inertia) of the motor apparatus (e.g. arm) and physical objects with which it interacts, as well as by the physical laws of motion. Adaptation to changing movement dynamics is an important aspect of motor learning, which we will refer to as dynamic motor adaptation.

The initial studies of dynamic motor adaptation altered the dynamics of reaching movements with novel velocity-dependent forces. Lackner and DiZio (1994) used velocity-dependent inertial forces (Coriolis forces) created by rotating the room in which the subjects performed the task. Shadmehr and Mussa-Ivaldi (1994) used velocity-dependent mechanical forces (curl forces) generated by a robotic arm held by the subject. These studies analyzed movement kinematics (e.g. arm position and velocity) to quantify performance before, during, and after the perturbation. A robust finding across these and subsequent studies was that subjects adapt to altered dynamics such that their performance is nearly identical in altered and normal (i.e. control) dynamic conditions. Building upon this basic psychophysical result, in this chapter we summarize two studies that were designed to investigate the involvement of motor cortical areas in this type of motor learning.

A broad survey of the neural structures involved in dynamic motor adaptation has emerged from studies of humans with specific nervous system lesions and through functional imaging and transcranial magnetic stimulation (TMS) in neurologically-intact individuals. Many of these studies have applied a common behavioral paradigm: subjects make reaching movements while holding onto a planar, two-link robotic arm which applies velocity-dependent forces. These forces are typically perpendicular to the hand's movement direction, giving rise to a curl force field (Shadmehr \& Mussa-Ivaldi, 1994). Adaptation to the curl field is profoundly impaired in patients with global cerebellar degeneration (Maschke et al., 2004; Smith \& Shadmehr, 2005) or with lesions to the cerebellar thalamus (Chen et al., 2006). However, adaptation is not impaired by striatal dysfunction, at least as manifest in Huntington's disease (Smith \& Shadmehr, 2005). A recent fMRI study has confirmed that the cerebellum, but not the striatum, is involved in curl field adaptation (Diedrichsen et al., 2005). This imaging study also found that adaptation involved cerebral cortical structures, including primary motor and somatosensory cortices and posterior parietal cortex (PPC). The latter cortical area has also been investigated by Della-Maggiore et al. (2004), who found that single-pulse TMS applied over the PPC after movement onset impaired adaptation to a curl field relative to controls. Therefore, the evidence from the human literature suggests that dynamic motor adaptation involves a cerebello-thalamocortical circuit. Our two experiments explore the cortical component of this circuit.

\section{Experiment I: Dynamic Motor Adaptation and Its Cortical Correlates}

To gain greater insight into the circuit governing dynamic motor adaptation, we turned to intracranial neural recordings in non-human primates. Dynamicsrelated neuronal activity has previously been found throughout motor areas of 
the cerebral cortex and cerebellum (Evarts, 1968; Humphrey et al., 1970; Thach, 1978; Cheney \& Fetz, 1980; Kalaska et al., 1989), but in behavioral tasks requiring minimal adaptation to applied loads. These studies suggest that kinetic parameters (e.g. joint torque) may be represented in motor cortex and the cerebellum, but do not tell us how these areas are involved in adaptation to novel dynamic conditions.

Our group has performed a series of studies investigating how motor areas of the cerebral cortex are involved in this type of motor learning (Gandolfo et al., 2000; Li et al., 2001; Padoa-Schioppa et al., 2002, 2004; Xiao et al., 2006). The paradigm was similar to the human studies: rhesus macaques were trained to make reaching movements in eight directions radially from a center target while holding onto a planar robotic arm that imposed curl force fields (see Methods; Fig. 1a,b). Like humans, the monkeys readily adapted to the curl field. Simultaneous single-unit recordings were performed in four different motor areas of the cerebral cortex (primary motor cortex, M1; supplementary motor area, SMA; and dorsal and ventral premotor cortex, PMd, PMv) before (baseline epoch; 160 trials), during (test epoch; 160 trials), and after (washout epoch; $160+$ trials) exposure to the force field (Fig. 2a). In control sessions, the curl field was not turned on in the test epoch (Fig. 2a). Neural activity was characterized by how the action potential discharge rate (i.e. firing rate) modulated with the eight movement directions (Fig. 1c). Cells were classified based on how this directional tuning changed between the three epochs of the task.

Examples of three classes of cells are show in Fig. 3. For each cell $(\mathbf{a}, \mathbf{b}$, and $\mathbf{c})$, a moving average of the instantaneous firing rate (left plot), instantaneous tuning depth (middle), and instantaneous preferred direction (right) is shown for each time-point in a $1.2 \mathrm{~s}$ window aligned on movement onset (horizontal axis) and for each trial throughout the recording session (vertical axis). The tuning depth and preferred direction were defined as the magnitude and direction of the resultant vector obtained by summing the firing rate-weighted vectors across the eight movement directions. Note that preferred direction, which is a circular variable, is shown relative to the grand mean preferred direction of the cell across all times and trials to minimize wrap-around effects. All three cells show movement-related activity that is either tonic (Fig. 3a, left plot) or phasic (Fig. 3b,c, left), beginning $\sim 100 \mathrm{~ms}$ before movement onset. All three cells also exhibit a transient increase in tuning depth around movement onset (Fig. 3, middle plots) and are not significantly tuned (as indicated by the black color) before or after this period.

The distinguishing feature of each cell's activity for the purposes of this experiment, however, is the change in tuning between epochs (i.e. between different dynamic conditions). In Fig. 3a, the cell exhibits no abrupt changes in average firing rate, tuning depth, or preferred direction between epochs. Similarly, there was no significant across-epoch change in preferred direction during the movement window for $34 \%$ of M1 cells $(\mathrm{N}=64), 52 \%$ of SMA cells $(\mathrm{N}=117), 38 \%$ of PMd cells $(\mathrm{N}=37)$, and $73 \%$ of PMv cells $(\mathrm{N}=26)$. Thus 


\section{a}
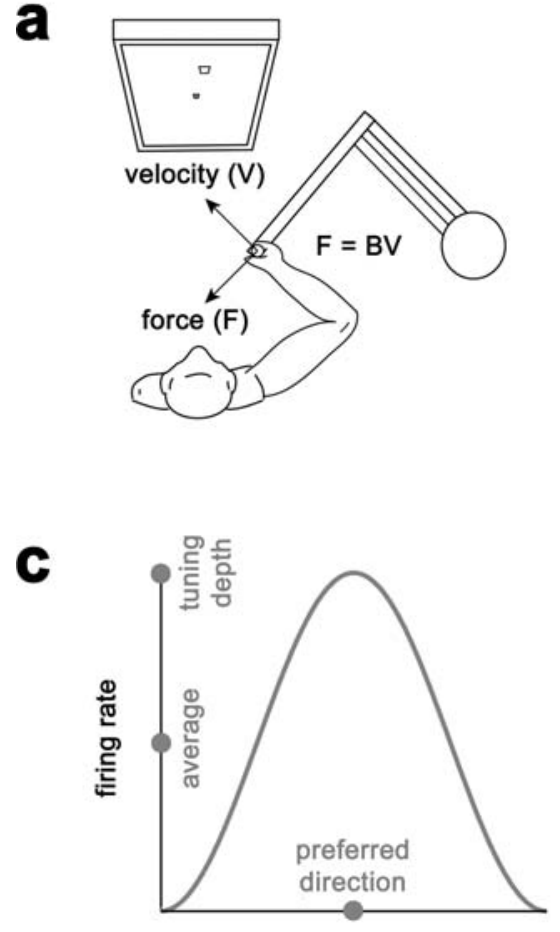

movement direction
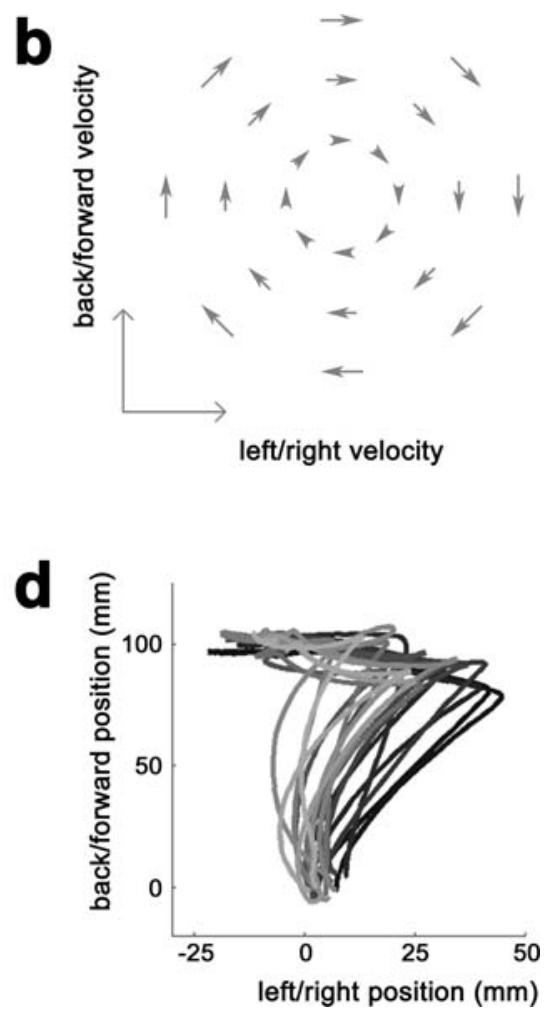

Fig. 1 Behavioral methods a Subjects (monkey or human) grasped a two-link robotic manipulandum with their right hand and used it to direct a cursor between targets shown on a computer monitor. The manipulandum was used to apply a force field, denote $\mathrm{B}$, to the hand (see Methods). b The specified forces were proportional and perpendicular to the hand velocity vector, resulting in a curl force field (e.g. clockwise, as shown). c Three parameters of the relationship between firing rate and movement direction (average firing rate, tuning depth, and preferred direction) were used to quantify neuronal activity in Experiment I. d Sample forward-directed trajectories of a participant experiencing a clockwise force field were deviated in the direction of the field, but more so at the beginning of the epoch (dark grey) than at the end (light grey). This force field adaptation was well-characterized by several related measures, including the signed peak perpendicular deviation used in Experiment II. (Panel d adapted with permission from Richardson et al., 2006; copyright 2007 by the Society for Neuroscience.)

the cell shown in Fig. 3a is typical of a class of cells which we refer to as "kinematic," since it is correlated to the relatively unchanging kinematics in each epoch but not the changing dynamics.

In contrast, there is an abrupt change in all three activity parameters at the epoch transitions for the cell in Fig. 3b. The phasic increase in average firing rate and tuning depth around movement onset has a longer duration in the test epoch compared to the baseline and washout epochs (Fig. 3b, left and middle plots). Also there is a large change in preferred direction during the test epoch 


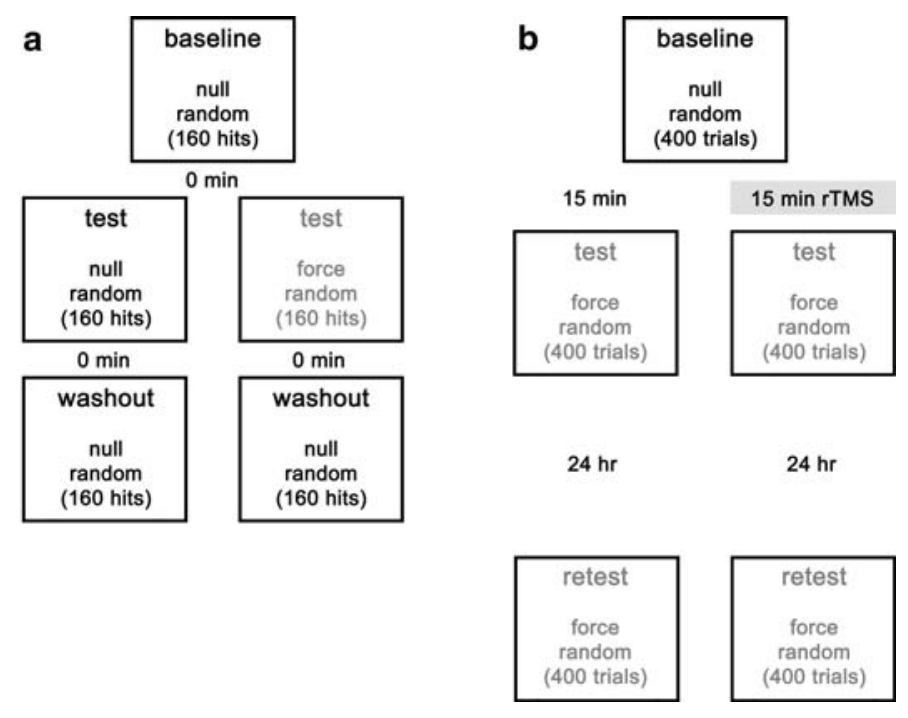

Fig. 2 Experimental designs Subjects experienced the following sequence of trial epochs (boxes) and breaks. Time increases in the downward direction. a In Experiment I, monkey subjects first performed a "baseline" epoch with null forces and random targets. This was immediately followed by a "test" epoch with either the null field or a force field, before the monkeys completed a final repeat of the baseline epoch referred to as a "washout" epoch. In each epoch the monkeys were required to complete 160 correct trials, i.e. "hits". The same monkeys experienced either of the conditions defined by the columns but on different days, and the cells in each of the two conditions were distinct. b In Experiment II, human subjects similarly performed three epochs beginning with a null-force, random-target baseline epoch. Subjects were expected to complete more trials than in Experiment I, although epoch lengths here were defined in terms of trial and not hit numbers. In contrast to Experiment I, all subjects experienced clockwise forces in the subsequent two epochs, and the last epoch was a second test (or "retest") epoch that followed the first test epoch after a 24-hour interval. Furthermore, half of the subjects in this experiment received rTMS to M1 in the interval between the baseline and test epoch. Conditions contained different participants (rather than different cells of the same subjects as in Experiment I)

that lasts $\sim 200 \mathrm{~ms}$ before movement onset until the end of movement (Fig. 3b, right). In this example, the preferred direction is more positive during the test epoch (as indicated by the grayscale reference bar). Note, however, that the instantaneous preferred direction profiles in the baseline and washout epochs are nearly identical. This pattern of preferred direction shifts - shifting $+\mathrm{x}$ degrees between baseline and test epoch and $-\mathrm{x}$ degrees from test to washout-was seen in $22 \%$ of M1 cells, $17 \%$ of SMA cells, $43 \%$ of $\mathrm{PMd}$ cells, and $12 \%$ of PMv cells. We refer to these cells as "dynamic," since they are correlated with the changing dynamic conditions (normal-altered-normal) of the task.

A third class of cell is show in Fig. 3c. The average firing rate changes very little over the course of the session (Fig. 3c, left plot). However, the cell abruptly 
a average (spikes/s)

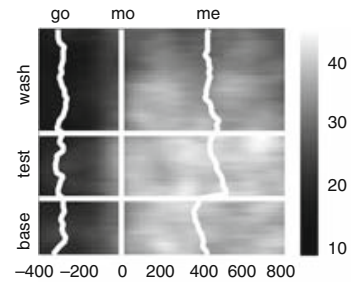

tuning depth (spikes/s)

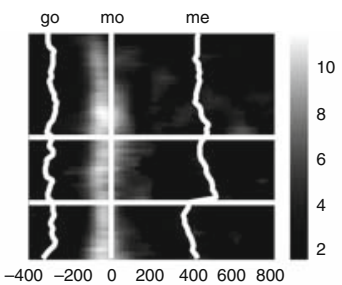

preferred direction $(\Delta \mathrm{deg})$

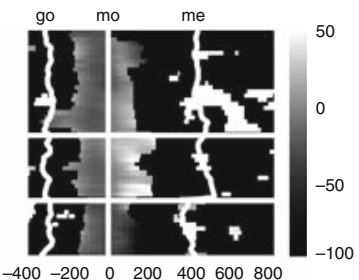

b
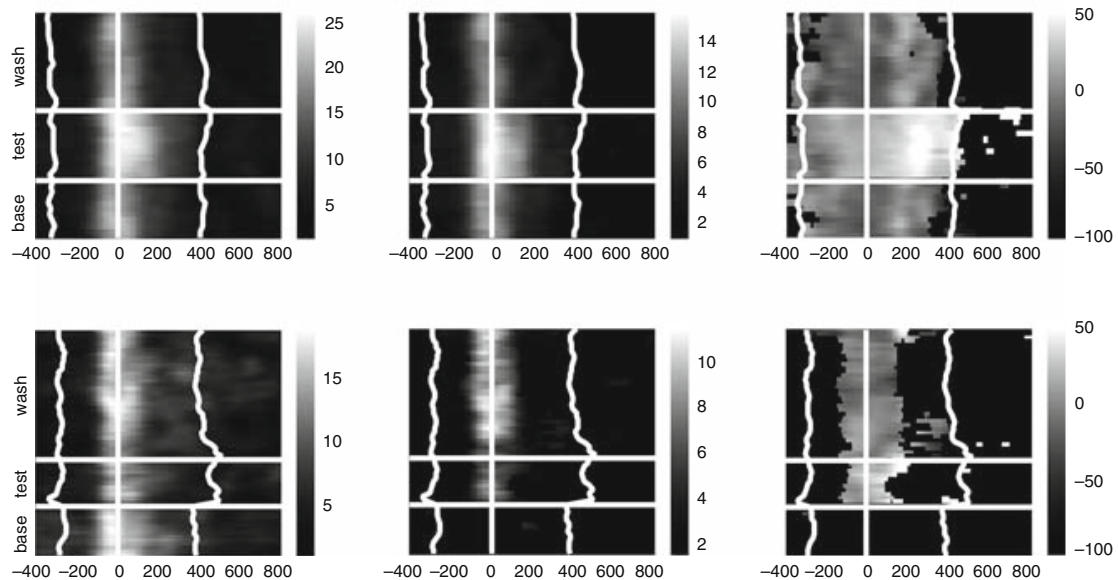

time (ms)

Fig. 3 Neuronal activity in motor cortex is modulated when learning novel movement dynamics (Experiment I) a "Kinematic" cell in the supplementary motor area (SMA). Instantaneous average firing rate (left plot), instantaneous tuning depth (middle plot), and instantaneous preferred direction (right plot) are shown. Trials are aligned on movement onset (mo). The time of the "go" signal (go) and end of movement (me) are also indicated by white lines. Black color on middle and right plots indicates times when the direction tuning was not significant ( $\mathrm{p}>0.01)$. Preferred directions are relative to 264 degrees. b "Dynamic" cell in dorsal premotor cortex (PMd). Preferred directions are relative to 52 degrees. c "Memory" cell in SMA. Preferred directions are relative to 186 degrees

becomes directionally tuned at the transition from baseline to test epoch and this tuning, in terms of both strength and preferred direction, remains largely unchanged throughout the remainder of the recording session (Fig. 3c, middle and right). This and similar types of maintained shifts in preferred direction throughout the washout epoch were seen in $41 \%$ of M 1 cells, $28 \%$ of SMA cells, $16 \%$ of PMd cells, and $12 \%$ of PMv cells. Since these cells have a change in activity specific to the altered dynamics that is maintained even after a return to the normal dynamics, we refer to them as "memory" cells.

These results confirm that the motor areas of the cerebral cortex play an important role in dynamic motor adaptation. More careful analysis of the 
pattern of activity changes may provide a more detailed description of what this role is and if it varies between cortical areas. For instance, the baseline fractions of kinematic, dynamic, and memory cells expected by chance remains to be established for control cell populations recorded on days without novel dynamics (Padoa-Schioppa et al., 2004). Nevertheless, an intriguing possibility raised by this experiment is that the motor cortex, including M1, may be involved not just in execution, but also in the memory of novel mechanical contexts. Most previous accounts have restricted this latter function to the cerebellum, based on evidence from functional imaging studies (Shadmehr \& Holcomb, 1997; Krebs et al., 1998). Our results, using more sensitive invasive recordings, suggest the plasticity underlying learning and memory of new dynamics may be more distributed throughout the cerebello-thalamo-cortical circuit.

\section{Experiment II: Dynamic Motor Adaptation and MI Disruption by rTMS}

In our second Experiment, we tested the role of human M1 in the learning and memory of novel dynamic environments, using a technique allowing causal rather than correlational inference. We employed low-frequency (1-Hz) repetitive TMS (rTMS) to interfere with M1 function while participants learned to make center-out and out-center movements in a clockwise force field (Fig. 1b). Based on prior literature, we expected that a 15-min train of subthreshold 1-Hz rTMS would be followed by a transient decrease in M1 excitability that would span the duration of the test epoch (Chen et al., 1997; Romero et al., 2002; Gangitano et al., 2002). Therefore, between the 400-trial baseline and test epochs the rTMS participants received $15 \mathrm{~min}$ of rTMS over M1 at a site previously localized as that from which biceps brachii contractions could most readily be evoked by TMS. (Control subjects rested for an equivalent time.) In the test epoch, all participants were introduced to the clockwise force field. This epoch was repeated 24 hours later as a retest epoch (Fig. 2b).

Prior to receiving rTMS, the rTMS group exhibited stable performance in the latter baseline epoch, as did the control group: there were no significant effects of Time or rTMS (Fig. 4a, left plot). In the test epoch, subjects exhibited a typical pattern of adaptation to the clockwise force field (Fig. 4a, middle), as revealed by a significant effect of Time $\left(\mathrm{F}_{(23,322)}=24.48, \mathrm{p}<0.0001\right)$. That is, reaching trajectories recorded under clockwise field conditions were initially deviated in the clockwise direction, but with experience they became gradually less deviated (Fig. 1d).

It may appear surprising that rTMS had no measurable effect on adaptation to the force field in the test epoch (Fig. 4a, middle plot). M1 excitability changes like those imposed on the rTMS subjects during the test epoch have certainly 

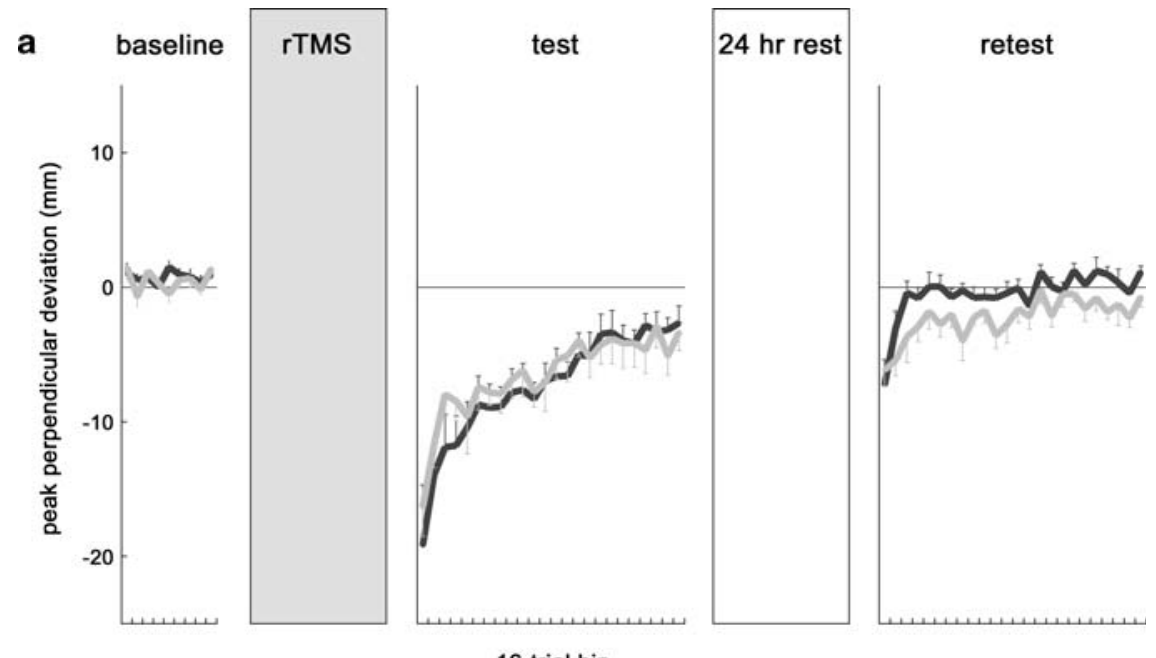

b

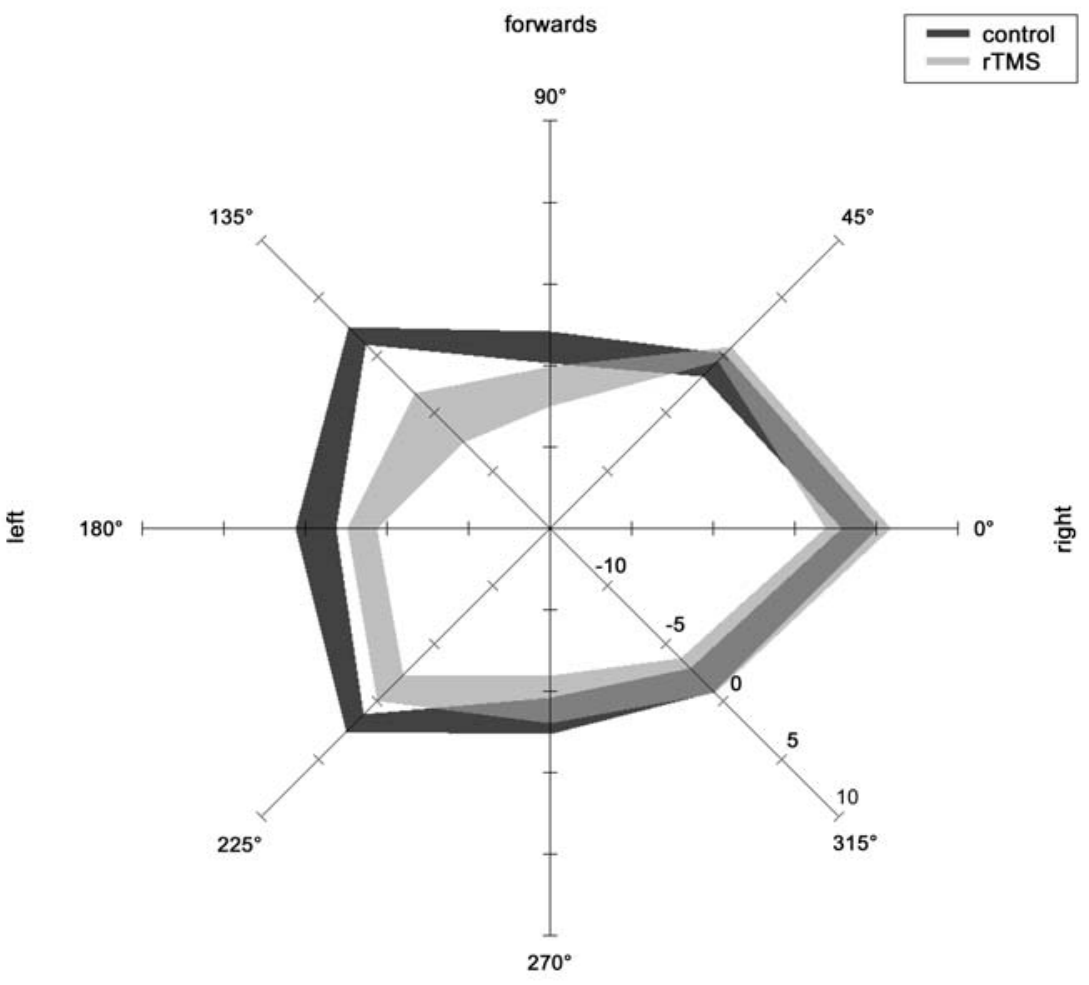

backwards

Fig. 4 rTMS of M1 spares initial motor performance and dynamic adaptation but impairs retest performance (Experiment II) a The performance of control (dark grey) and rTMS 
been shown to measurably affect behavior (Pascual-Leone et al., 1994). Given this, the comparable performance level and rate of learning of the rTMS and control subjects in the test epoch imply that M1 is either acutely redundant with other brain areas (Lee et al., 2003), or that it is not involved in motor execution and initial adaptation (Diedrichsen et al., 2005; Paz et al., 2005). The latter possibility seems unlikely given the presence of dynamically modulated cells within M1, as reported in Experiment I. However, that experiment also found that other cortical motor areas possessed a comparable breakdown of functional cell types, indicating that compensatory cortical control of these movements by areas outside of M1 may explain the unimpaired adaptation of the rTMS participants. And indeed, M1 has previously been shown to be noncritical for motor performance and early motor learning in both force field learning and ballistic movement tasks (Muellbacher et al., 2002; Baraduc et al., 2004).

The truly novel result of Experiment II came in the retest epoch 24 hours after initial learning (Fig. 4a, right plot). At retest, participants once again demonstrated adaptation to the forces, as reflected in a significant main effect of Time $\left(\mathrm{F}_{(23,322)}=8.33, \mathrm{p}<0.0001\right)$. But in contrast to the learning epoch, the rTMS subjects now performed with greater kinematic error than controls: there was a significant main effect of $\operatorname{rTMS}\left(\mathrm{F}_{(1,14)}=4.30, \mathrm{p}<0.05\right)$. Both groups exhibited some memory retention at retest since initial retest errors were smaller than early test-epoch errors (Fig. 4a, right). Between the first third of the test and retest epochs, for instance, there was a significant effect of Time $\left(F_{(1,14)}=100.67\right.$, $\mathrm{p}<0.0001)$. But these savings were present in different degrees between the two groups, as shown by a significant $\mathrm{rTMS} \times$ Time interaction in the same comparison $\left(\mathrm{F}_{(1,14)}=6.47, \mathrm{p}<0.01\right)$. Not only were these memory savings present, but (beyond the first few trials) the initial retest trajectories were less deviated even than those late in the test epoch, at least for control subjects. Thus in comparing the last third of the test epoch with the first third of the retest, significant Time and $\mathrm{rTMS} \times$ Time effects were again evident $\left(\mathrm{F}_{(1,14)}=8.36, \mathrm{p}<0.01\right.$ and $\mathrm{F}_{(1,14)}=3.54, \mathrm{p}<0.05$, respectively).

In a further unexpected finding, this relative memory impairment of the rTMS subjects at retest was not uniform over all movement directions (Fig. 4b), but

Fig. 4 (continued) (light grey) groups, as measured by signed peak perpendicular deviation, is shown for late baseline test and retest trials (mean \pm S.E.M. of 16-trial bins; lower error bar for control group and upper error bar for rTMS group are removed for clarity). b The relatively impaired retest performance of rTMS subjects was direction-specific. The average retest performance error for control and rTMS groups is shown for each movement direction (shaded region indicates \pm 1 S.E.M. around the mean performance over the entire epoch). The performance (shown on the radial axis) is quantified by the signed peak perpendicular deviation, where negative errors indicate clockwise deviation, as in panel a. (Panels adapted with permission from Richardson et al., 2006; copyright 2007 by the Society for Neuroscience.) 
was evident only in leftward-directed movements. A repeated measures ANOVA found significant effects not only of rTMS (indicating rTMS subjects' greater overall error) and movement direction (reflecting the nonuniformity of all subjects' error over directions) but also a significant rTMS $\times$ direction interaction $\left(\mathrm{F}_{(1,14)}=5.17, \mathrm{p}<0.05 ; \mathrm{F}_{(7,98)}=13.29, \mathrm{p}<0.0001 ;\right.$ and $\mathrm{F}_{(7,98)}=2.70, \mathrm{p}<0.05$, respectively). No such interaction effect was present in the test epoch (not shown). Curiously, this directional specificity of the rTMS subjects' retest error coincided with the tuning of the biceps brachii muscle (e.g. Fig. 7 of Thoroughman \& Shadmehr, 1999) - the same muscle we chose to target for consistency's sake among all rTMS subjects. Although the anatomical imprecision of TMS (e.g. Lee et al., 2003) makes it highly unlikely that our rTMS spared M1's representation of other muscles, it is nevertheless possible that rTMS may have had a relatively more pronounced effect in those movement directions that most involved the biceps.

The disruptive effects of rTMS were temporally circumscribed, as well, since cortical excitability is known to return to normal approximately 10 min following the end of stimulation (Romero et al., 2002). The implication that M1's role in motor memory formation begins at the time of acquisition is similar to the findings of Muellbacher et al. (2002). Using a ballistic finger movement task, they found that rTMS of M1 immediately after practice (not before, as in our experiment) interfered with subject's retention of the novel motor skill. But in contrast to the Muellbacher et al. (2002) results, our rTMS subjects did not return to naïve levels but instead retained their new motor skill (Fig. 4a). The impairment of the rTMS participants was only evident relative to controls (and only in leftward movement directions; Fig. 4b). The results of Baraduc et al. (2004) may help to explain the difference between our results and those of Muellbacher et al. (2002). They compared the effects of M1 disruption by rTMS following both ballistic movement practice (as in Muellbacher et al., 2002) and dynamic adaptation, and found a difference in the degree of skill retention following the disruption. But while confirming the results of Muellbacher et al. (2002) for ballistic movements, they found that M1 disruption following force field adaptation did not interfere with the retention of this learning.

Our results agree with both of these studies in revealing no role of M1 in stabilizing newly-acquired dynamic motor memories (since even rTMS subjects appeared to have retained their learning of the previous day). However, our experiment also implies that proper M1 function during dynamic adaptation enables offline skill improvement (since only control subjects had improved in performance between late test and early retest; Fig. 4a, right plot). Such offline improvement is the hallmark of "consolidation" as defined in procedural memory research (Robertson et al., 2004; Walker, 2005). Indeed, in a recent study by Robertson et al. (2005) rTMS of M1 following implicit sequence learning was associated with a disruption of off-line improvement (specifically, that which would normally accrue over daytime hours). 


\section{Summary}

What brain areas are critical for dynamic motor learning? The neuronal recordings of Experiment I implicate a network of motor cortical areas (and not just the cerebellum), including M1, PMd, PMv and SMA. These areas may each encode not only kinematic and dynamic parameters of movement, but also changes in activity that could serve as a "memory" of novel forces even after they are no longer present. Although the statistical significance of each of these distributed functional cell types remains to be assessed, it is possible that in MI, as in higher motor cortical areas, there is a population of cells involved not only in motor execution but in motor memory. Whatever the role of M1 in motor learning, Experiment II indicates that M1 is not critical to the network underlying motor adaptation. Disruption of human M1 excitability by rTMS was not associated with any simultaneous impairment of adaptation to a clockwise field. Instead, the relative retest performance deficit of the rTMS subjects suggests that M1 may be uniquely important for enabling offline skill improvement following initial adaptation.

\section{Methods}

Experiment I involved six rhesus macaques (Macaca mulatta). Experiment II involved right-handed, college-age students, screened for history of seizures, epilepsy, head injuries, neurological illness, and use of psychoactive medications. In this Experiment 8 each of the 16 subjects were randomly assigned to two experimental groups ("control" and "rTMS").

\section{Behavioral paradigm}

A custom two-link, planar robotic manipulandum was used in Experiment II (Fayé, 1983); Experiment I involved a monkey-scale version of the same apparatus (Fig. 1a). The manipulandum provided a continuous record of the subject's hand kinematics as well as a mechanism with which to apply forces to the arm. Subjects were trained (Experiment I) or instructed (II) to hold onto the manipulandum with their right hand and make reaching movements to targets presented on a monitor (for details, see Shadmehr and Mussa-Ivaldi, 1994). The targets were white squares of width $14-17 \mathrm{~mm}$ (Experiment I) or $10 \mathrm{~mm}$ (II) appearing on a black screen. Motions of the handle were represented on the screen as continuous movements of a 3-mm-wide square (Experiment I) or an 8-mm-wide crosshair (II).

The movement distances were $10 \mathrm{~cm}$ both in real space and as shown on the monitor. In Experiment I, the targets included eight peripheral squares spaced uniformly around a central square. We focused only on the "center-out" 
movements, which defined eight movement directions. In Experiment II, the targets included four peripheral squares circumscribing half a circle around a central square, such that center-out and "out-center" movements (both of which we considered) again resulted in eight movement directions uniformly spanning $360^{\circ}$.

In Experiment I, monkeys were required to: (1) maintain the cursor within the central square for an initial $1 \mathrm{~s},(2)$ wait a variable time $(1.0 \pm 0.5 \mathrm{~s})$ for a movement cue, i.e. the disappearance of the center square, (3) move the cursor to a peripheral target square in less than $2 \mathrm{~s}$ without deviating from the ideal movement direction by more than $\pm 60^{\circ}$, and (4) hold the cursor within the peripheral target for $1 \mathrm{~s}$. Participants in Experiment II were subject to fewer constraints. They were given $0.50 \pm 0.05 \mathrm{~s}$ to complete each movement, starting from the time at which they left the origin square, and lasting until their cursor entered the target. Upon acquiring the target, a further 0.5-s within-target hold time had to be observed in order for the trial to be a "success". In Experiment II, the target remained on the screen even if subjects failed to reach it in the allotted time - that is, all trials, including hits and misses, had to be completed. Trials completed according to the specified time constraints were indicated to the subject by liquid reward (Experiment I) or a brief sound (II). Trials completed too quickly or too slowly in Experiment II were indicated to the subject by a transition in the target color from white to red. Successful trials are referred to here as "hits". The targets were presented pseudorandomly (with constraints on the relative frequency of different movement directions).

In Experiment I, monkeys performed three epochs of 160 hits (not trials) in each session, with no break in between (Fig. 2a). The epochs were denoted "baseline," "test," and "washout". In Experiment II, participants experienced three epochs in a single session: a baseline (two subepochs of 250 trials and 150 trials separated by $15 \mathrm{~min}$ ), test (400 trials), and "retest" (400 trials) epoch (Fig. 2b). The duration of the interval between the baseline and test epochs was approximately $15 \mathrm{~min}$; the test and retest epochs were separated by $24 \mathrm{hr}$.

In each experiment, subjects experienced a null $(0 \mathrm{~N} \cdot \mathrm{s} / \mathrm{m})$ force field during the baseline and (in Experiment I) washout epochs (Fig. 2). On control days in Experiment I, subjects also received a null field during the test epoch. No forces were applied by the manipulandum motors during these epochs. Otherwise, during the test and retest epochs the motors generated a velocity-dependent force field (Fig. 1b). Forces were calculated on-line as $f=\mathbf{B} \dot{x}$, where $\mathrm{B}=\left[\begin{array}{cc}0 & b \\ -b & 0\end{array}\right]$ and $\dot{x}$ was the movement velocity. The force field gain factor $b$ was set to $\pm 6 \mathrm{~N} \cdot \mathrm{s} / \mathrm{m}$ (Experiment I) or $+15 \mathrm{~N} \cdot \mathrm{s} / \mathrm{m}$ (II). In Experiment I the forces in some sessions were counterclockwise instead of clockwise.

\section{Electrophysiology}

In Experiment I, after a monkey was sufficiently trained on the task, a headrestraining device was fixed to the skull. The monkey was then re-trained to 
perform the task under head-fixed conditions. Then a craniotomy was performed, leaving the dura mater intact, and a recording well was implanted. The well was located over primary motor cortex in the first pair of monkeys, over supplementary motor cortex in the second pair, and over premotor cortex in the third pair. About a week following implantation of the recording well, an intracortical microstimulation (ICMS) study was conducted over the course of several days to map out the proximal arm representation in the cortical area. ICMS was delivered via a $\sim 300 \mathrm{k} \Omega$ impedance microelectrode using a train of 20 biphasic pulses at $330 \mathrm{~Hz}$ and $10-40 \mu \mathrm{A}$. Stimulus-evoked muscle twitches were observed and mapped to the location of the stimulus. After locating the proximal arm representations, extracellular recordings were made from these locations during each session that the monkeys performed the task. For the recordings, epoxylite-insulated tungsten microelectrodes were used, with 1-3 $\mathrm{M} \Omega$ impedance and $250 \mu \mathrm{m}$ diameter shaft tapered down to a $3 \mu \mathrm{m}$ diameter tip. The electrodes were lowered using a manual microdrive with a depth resolution of approximately $30 \mu \mathrm{m}$. Due to dimpling of the cortex upon penetration and limitations in depth resolution, the laminar location of the recorded cortical cells was generally not known. Up to eight recording electrodes were used in each session. The analog electrical signals from the electrodes were passed to a preamplifying headstage $(1 \times$ gain) located about $5 \mathrm{~cm}$ from the electrodes and then to an amplifier, where they were amplified $(10000 \times)$ and filtered with a passband of $300 \mathrm{~Hz}$ to $10 \mathrm{kHz}$. Action potentials were detected by a manuallydetermined threshold crossing and the spike times, along with behavioral task event times, were saved for off-line analysis. Spike waveforms were digitized ( $1.75 \mathrm{~ms}$ duration, 16 bit resolution at $50 \mathrm{kHz}$ ) and saved for subsequent clustering analysis.

\section{Transcranial magnetic stimulation}

In Experiment II, we delivered TMS using a Magstim Super Rapid stimulator (Whitland, Wales, UK). Prior to using repetitive TMS to interfere with M1 function in the rTMS subjects, we first applied single pulses of TMS over the left motor cortex of these participants in order to identify the location from which visible contractions of the right biceps brachii muscle could be reliably induced (i.e. on at least 5 of 10 consecutive attempts) with minimal stimulator intensity (but at least $50 \mu \mathrm{V}$ ). This localization procedure was performed during a break after the first 250 trials of the baseline epoch. The $15 \mathrm{~min}$ of $1 \mathrm{~Hz}$ rTMS were then administered at the target location between the baseline and test epochs, at $90 \%$ of the intensity needed to evoke biceps contraction by the above localization - a subthreshold intensity level that previous research has associated with a long-lasting depression of motor cortex excitability (Chen et al., 1997; Romero et al., 2002; Gangitano et al., 2002). rTMS pulses were delivered using a double $70 \mathrm{~mm}$ hand-held figure-of-eight coil (Magstim), placed tangentially to the 
scalp in a $45^{\circ}$ posterior-to-anterior and lateral-to-medial orientation. Because the rTMS was delivered prior to force field exposure - while all subjects were resting "offline" - no sham stimulation was used in the control participants. Had the TMS been delivered during learning, it might more easily have been the cause of non-specific attentional or other behavioral effects (Robertson et al., 2003).

\section{Analysis}

In Experiment I we focused our analysis on the neural activity accompanying learning. Neurons were characterized by how their firing rate modulated with movement direction (Fig. 1c). Tests for significance of directional tuning and significance of shifts in directional tuning are described in detail in Li et al. (2001). The plots in Fig. 3 were generated by first convolving a cell's spike train on each trial with a Gaussian kernel ( $40 \mathrm{~ms}$ standard deviation) to estimate the instantaneous firing rate. Then, for each millisecond along this instantaneous firing rate, tuning curves were computed and significance of tuning tested using a permutation test $(\mathrm{p}<0.01)$. When significant, tuning depth and preferred direction were determined (Fig. 1c). A moving average of all three parameters (average firing rate, tuning depth, and preferred direction) was then computed across trials (40 trial bin size, 8 trial step size) for each epoch separately. Plots of preferred direction are in terms of change in direction relative to the grand mean preferred direction of the cell across all times and trials, to minimize wraparound effects.

In Experiment II, we quantified performance on each trial as the peak perpendicular deviation relative to a straight line connecting the beginning and end positions of the trial (e.g. Shadmehr \& Moussavi, 2000; Donchin et al., 2002; Karniel \& Mussa-Ivaldi, 2003). This measure was "signed" in that clockwise deviations were scored as positive and counterclockwise deviations negative. We obtained qualitatively similar results with other, related measures of trajectory deviation such as deviation area.

In Experiment II, trials in which the subject failed to reach the target in the allotted time limits were still included in the analysis. However, we rejected any trial in which the movement time was outside a $0.50 \pm 0.25$-s time window. Trials were binned by 16 trials in each epoch and condition. Statistical results were based on the within-bin-averaged trajectory error. Aftereffects, i.e. changes in performance error following the test epoch, were estimated by comparing the mean scores of the final eight trial bins in the test epoch and the first eight trial bins in the retest epoch. Main and interaction effects of rTMS, movement Direction, and Time on trajectory error were assessed using repeated measures ANOVAs. All significant effects at the $\mathrm{p}<0.05$ level are reported. 
Acknowledgments We thank Margo Cantor for technical assistance in Experiment I, and Daniel Press, Camillo Padoa-Schioppa, and Antoni Valero-Cabré for collaboration in Experiment II. The work in Experiment I was supported by a National Institute of Neurological Disorders and Stroke grant (NINDS NS044393) and a National Institutes of Health grant (NIH MH48185) to Emilio Bizzi. The work in Experiment II was supported by a National Institutes of Health grant (NIH K23-MH65434) to Daniel Press. Figure 1d and panels of Fig. 4 are adapted with permission from Richardson et al. (2006).

\section{References}

Baraduc P, Lang N, Rothwell JC, Wolpert DM (2004) Consolidation of dynamic motor learning is not disrupted by rTMS of primary motor cortex. Curr Biol 14: 252-256.

Chen H, Hua SE, Smith MA, Lenz FA, Shadmehr R (2006) Effects of human cerebellar thalamus disruption on adaptive control of reaching. Cereb Cortex 16: 1462-1473.

Chen R, Classen J, Gerloff C, Celnik P, Wassermann EM, Hallett M, Cohen LG (1997) Depression of motor cortex excitability by low-frequency transcranial magnetic stimulation. Neurology 48: 1398-1403.

Cheney PD, Fetz EE (1980) Functional classes of primate corticomotoneuronal cells and their relation to active force. J Neurophysiol 44: 773-791.

Della-Maggiore V, Malfait N, Ostry DJ, Paus T (2004) Stimulation of the posterior parietal cortex interferes with arm trajectory adjustments during the learning of new dynamics. J Neurosci 24: 9971-9976.

Diedrichsen J, Hashambhoy Y, Rane T, Shadmehr R (2005) Neural correlates of reach errors. J Neurosci 25: 9919-9931.

Donchin O, Sawaki L, Madupu G, Cohen LG, Shadmehr R (2002) Mechanisms influencing acquisition and recall of motor memories. J Neurophysiol 88: 2114-2123.

Evarts EV (1968) Relation of pyramidal tract activity to force exerted during voluntary movement. J Neurophysiol 31: 14-27.

Fayé IC (1983) An impedance controlled manipulandum for human movement studies. M.S. Thesis. Cambridge, MA: MIT Press.

*Gandolfo F, Li CR, Benda BJ, Padoa-Schioppa C, Bizzi E (2000) Cortical correlates of learning in monkeys adapting to a new dynamical environment. Proc Natl Acad Sci USA 97: $2259-2263$.

Gangitano M, Valero-Cabré A, Tormos JM, Mottaghy FM, Romero JR, Pascual-Leone A (2002) Modulation of input-output curves by low and high frequency repetitive transcranial magnetic stimulation of the motor cortex. Clin Neurophysiol 113: 1249-1257.

Humphrey DR, Schmidt EM, Thompson WD (1970) Predicting measures of motor performance from multiple cortical spike trains. Science 170: 758-762.

Kalaska JF, Cohen DA, Hyde ML, Prud'homme M (1989) A comparison of movement direction-related versus load direction-related activity in primate motor cortex, using a two-dimensional reaching task. J Neurosci 9: 2080-2102.

Karniel A, Mussa-Ivaldi FA (2003) Sequence, time, or state representation: how does the motor control system adapt to variable environments? Biol Cybern 89: 10-21.

Krebs HI, Brashers-Krug T, Rauch SL, Savage CR, Hogan N, Rubin RH, Fischman AJ, Alpert NM (1998) Robot-aided functional imaging: application to a motor learning study. Hum Brain Mapp 6: 59-72.

Lackner JR, DiZio P (1994) Rapid adaptation to Coriolis force perturbations of arm trajectory. J Neurophysiol 72: 299-313.

Lee L, Siebner HR, Rowe JB, Rizzo V, Rothwell JC, Frackowiak RS, Friston KJ (2003) Acute remapping within the motor system induced by low-frequency repetitive transcranial magnetic stimulation. J Neurosci 23: 5308-5318. 
Li CR, Padoa-Schioppa C, Bizzi E (2001) Neuronal correlates of motor performance and motor learning in the primary motor cortex of monkeys adapting to an external force field. Neuron 30: 593-607.

Maschke M., Gomez CM, Ebner TJ, Konczak J (2004) Hereditary cerebellar ataxia progressively impairs force adaptation during goal-directed arm movements. J Neurophysiol 91: 230-238.

*Muellbacher W, Ziemann U, Wissel J, Dang N, Kofler M, Faccini S, Boroojerdi B, Poewe W, Hallett M (2002) Early consolidation in human primary motor cortex. Nature 415: 640-644.

Padoa-Schioppa C, Li CR, Bizzi E (2002) Neuronal correlates of kinematics-to-dynamics transformation in the supplementary motor area. Neuron 36: 751-765.

Padoa-Schioppa C, Li CR, Bizzi E (2004) Neuronal activity in the supplementary motor area of monkeys adapting to a new dynamical environment. J Neurophysiol 91: 449-473.

Pascual-Leone A, Grafman J, Hallett M (1994) Modulation of cortical motor output maps during development of implicit and explicit knowledge. Science 263: 1287-1289.

Paz R, Natan C, Boraud T, Bergman H, and Vaadia E (2005) Emerging patterns of neuronal responses in supplementary and primary motor areas during sensorimotor adaptation. J Neurosci 25: 10941-10951.

*Richardson AG, Overduin SA, Valero-Cabré A, Padoa-Schioppa C, Pascual-Leone A, Bizzi E, Press DZ (2006) Disruption of primary motor cortex prior to learning impairs memory of movement dynamics. J Neurosci 26: 12466-12470.

*Robertson EM, Pascual-Leone A, Miall RC (2004) Current concepts in procedural consolidation. Nat Rev Neurosci 5: 1-7.

Robertson EM, Press DZ, Pascual-Leone A (2005) Off-line learning and the primary motor cortex. J Neurosci 25: 6372-6378.

Robertson EM, Theoret H, Pascual-Leone A (2003) Studies in cognition: the problems solved and created by transcranial magnetic stimulation. J Cogn Neurosci 15: 948-960.

Romero JR, Anschel D, Sparing R, Gangitano M, Pascual-Leone A (2002) Subthreshold low frequency repetitive transcranial magnetic stimulation selectively decreases facilitation in the motor cortex. Clin Neurophysiol. 113: 101-107.

Sanes JN, Donoghue JP (2000) Plasticity and primary motor cortex. Annu Rev Neurosci 23: 393-415.

*Shadmehr R, Holcomb HH (1997) Neural correlates of motor memory consolidation. Science 277: 821-825.

Shadmehr R, Moussavi ZMK (2000) Spatial generalization from learning dynamics of reaching movements. J Neurosci 20: 7807-7815.

*Shadmehr R, Mussa-Ivaldi FA (1994) Adaptive representation of dynamics during learning of a motor task. J Neurosci 14: 3208-3224.

Smith MA, Shadmehr R (2005) Intact ability to learn internal models of arm dynamics in Huntington's disease but not cerebellar degeneration. J Neurophysiol 93: 2809-2821.

Thach WT (1978) Correlation of neural discharge with pattern and force of muscular activity, joint position, and direction of intended next movement in motor cortex and cerebellum. J Neurophysiol 41:654-676.

Thoroughman KA, Shadmehr R (1999) Electromyographic correlates of learning an internal model of reaching movements. J Neurosci 19: 8573-8588.

Walker MP (2005) A refined model of sleep and the time course of memory formation. Behav Brain Sci 28: 51-104.

Xiao J, Padoa-Schioppa C, Bizzi E (2006) Neuronal correlates of movement dynamics in the dorsal and ventral premotor area in the monkey. Exp Brain Res 168: 106-119.

The references marked with an asterisk $(*)$ are specifically recommended for further introduction or background to the topic. 